\title{
Caracterização química de frutos de morangueiro cultivados em vasos sob sistemas de manejo orgânico e convencional
}

\section{Chemical characterization of strawberry fruits in the organic and conventional cropping systems in pots}

\author{
Letícia Kurchaidt Pinheiro Camargo ${ }^{1 *}$; Juliano Tadeu Vilela de Resende ${ }^{2}$; \\ Alexandre Gonçalves Galvão ${ }^{3}$; João Eduardo Baier; \\ Marcos Ventura Faria ${ }^{5}$; Cristhiano Kopanski Camargo ${ }^{6}$
}

\section{Resumo}

A qualidade nutricional de frutos de morango das cultivares Camarosa e Sweet Charlie, obtidos em sistemas orgânico e convencional, foi comparada com base na caracterização química dos frutos mediante análises dos teores de sólidos solúveis (SS), acidez titulável (AT), relação (SS/AT), umidade, açúcares redutores e açúcares totais. O delineamento experimental utilizado foi de blocos casualizados com quatro repetições. De modo geral foi observado efeito diferenciado dos tipos de sistemas de cultivo sobre a maioria das características e, principalmente, do comportamento das cultivares dentro de cada sistema. O sistema de produção convencional foi mais efetivo em aumentar os teores de SS, AT e SS/AT para a cultivar Sweet Charlie e o orgânico para a cultivar Camarosa. A umidade no sistema convencional foi significativamente superior ao orgânico para a cultivar Sweet Charlie. As porcentagens de açúcares totais no sistema convencional foram estatisticamente superiores quando comparadas ao orgânico, para a cultivar Sweet Charlie. Assim, verificou-se comportamento diferenciado das cultivares em relação ao sistema de cultivo, para a maioria das variáveis estudadas.

Palavras-chave: Fragaria $x$ ananassa, análise química, sistemas de produção

\begin{abstract}
The nutritional quality of strawberry fruits of the cultivars Camarosa and Sweet Charlie cultivated in organic and conventional cropping systems was compared based on chemical characterization of the fruits by analyses of soluble solids content (SS), titratable acidity (AT), ratio (SS/AT), humidity, reducer sugars and total sugars. The experiment was performed in completely randomized blocks design, with four replications. There were differentiated effects of the cropping systems on the most of the evaluated traits and on the behavior of cultivars in each cropping system. The conventional cropping system was more effective in increasing contents of SS, AT and SS/AT in cultivar Sweet Charlie and the organic cropping system in cultivar Camarosa. The humidity in the conventional cropping system was significantly higher than that obtained from the organic cultivar Sweet Charlie. The percentages of total sugars in the conventional cropping system were statistically higher when compared with the organic for cultivar Sweet Charlie. It was verified a differentiated behavior of the cultivars in relation to the cropping system, for the most of evaluated traits.
\end{abstract}

Key words: Fragaria $x$ ananassa, chemical analysis, cropping systems

\footnotetext{
Aluna de mestrado do Departamento de Agronomia, UNICENTRO; e-mail: lpinheiro@unicentro.br

2 Docente do Departamento de Agronomia, DEAGRO/UNICENTRO; e-mail: jresende@unicentro.br

3 Aluno de graduação do Departamento de Agronomia, UNICENTRO; e-mail: galvao_alexandre@hotmail.com

4 Aluno de graduação do Departamento de Agronomia, UNICENTRO; e-mail: joão_bayer@hotmail.com

5 Docente do Departamento de Agronomia, DEAGRO/UNICENTRO; e-mail: mfaria@unicentro.br

6 Aluno de mestrado do Departamento de Agronomia, UNICENTRO; e-mail: ccamargo@unicentro.br

* Autor para correspondência
} 


\section{Introdução}

A produção de morango no Brasil é estimada em torno de 100 mil toneladas em uma área aproximada de 3.500 ha, com destaque para as regiões sul e sudeste, sendo o estado de Minas Gerais o maior produtor. A cultura do morango é caracterizada pelo elevado contingente de mão-de-obra, apresentando substancial importância social e econômica, sendo geradora de emprego e renda, principalmente para comunidades de agricultores familiares (ANTUNES et al., 2007).

O morango produzido no Brasil e no mundo é em grande parte proveniente de cultivo em sistemas convencionais, caracterizado pelo uso intensivo de produtos químicos, podendo receber até 45 pulverizações com agrotóxicos durante o ciclo da cultura (DAROLT, 2003), haja vista a suscetibilidade das cultivares a uma vasta gama de doenças e pragas. Além disso, o sistema de cultivo do tipo convencional baseia-se no emprego exagerado de insumos externos, de fora da propriedade ou da região, geralmente de alto custo e que causam a dependência financeira, tecnológica e biológica do produtor. Há ainda que se considerar o impacto ambiental que o sistema convencional causa, com problemas de erosão, baixa produtividade das terras e culturas, produção de dejetos, efluentes ou resíduos que são considerados lixo, e são depositados diretamente na natureza (SAMINÊZ, 2000).

Nesse sentido, a conscientização sobre os riscos decorrentes do uso contínuo de agrotóxicos tem levado ao desenvolvimento e aperfeiçoamento do sistema de cultivo orgânico, permitindo aos agricultores colocar no mercado produtos com menores níveis de contaminação química e microbiológica, em consonância com a legislação vigente no país (SANHUEZA, 2000).

Estudos realizados pelo Conselho Nacional de Pesquisa dos Estados Unidos em 1989 indicaram que os sistemas alternativos de cultivo apresentam resultados financeiros favoráveis, diminuindo os custos de produção sem diminuir a produtividades e, em alguns casos, aumenta os rendimentos e a produtividade, fazendo uso reduzido de insumos químicos, atenuando os efeitos adversos da agricultura sobre o ambiente e à saúde humana, além de manter a boa aparência dos produtos (SAMINÊZ, 2000).

Segundo Darolt (2001), a prática de alguns produtores da região Sul do Brasil tem mostrado que existe viabilidade técnica, econômica, social e ecológica da produção orgânica de morangos. A adubação orgânica é importante não somente para o fornecimento de nutrientes às plantas, mas pela melhoria das condições físicas e biológicas do solo, contribuindo para um melhor aproveitamento dos nutrientes aplicados (SCHERER et al., 2003).

Vários fatores podeminfluenciarnas propriedades químicas e físicas de um produto agrícola: a cultivar utilizada, o tipo de solo e clima, o ano climático e o sistema de produção (orgânico ou convencional) (DAROLT, 2003). Segundo Williams (2002), existe um número limitado de estudos comparando as composições de nutrientes produzidos organicamente e convencionalmente, e poucos são os resultados conclusivos nas diversas culturas, sendo mais evidente quando se trata do morangueiro (SCHERER et al., 2003), principalmente com relação à qualidade nutricional.

Os estudos comparativos que focam a qualidade organoléptica estão ainda em estágio inicial e mostram resultados variáveis, o que não permite afirmar que existam diferenças significativas entre os diferentes sistemas (DAROLT, 2003). Sendo assim, a caracterização física e química dos frutos é de grande importância quando se estuda o comportamento de cultivares em diferentes ambientes, pois permite obter informações sobre a qualidade do produto final (DIAS et al., 2002).

O presente trabalho teve como objetivo caracterizar quimicamente frutos de cultivares de morangueiro produzidos em vasos sob manejo orgânico e convencional. 


\section{Material e métodos}

Foram utilizados frutos de duas cultivares de morango (Camarosa e Sweet Charlie), provenientes de dois sistemas de cultivo, orgânico e convencional. $\mathrm{O}$ experimento foi implantado no Setor de Olericultura da Universidade Estadual do CentroOeste(UNICENTRO) em Guarapuava/PR, em vasos mantidos sob casa de vegetação. Foram utilizadas mudas provenientes de matrizes, propagadas no viveiro no Setor de Olericultura da UNICENTRO. Cumpre ressaltar que a multiplicação das mudas ocorreu dentro do manejo convencional, porém utilizando o mínimo possível de insumos químicos.

As mudas que foram retiradas para plantio receberam "toalete", onde foram retiradas folhas velhas, secas e com possíveis sinais de doença. Após a toalete, as raízes ficaram imersas por 5 minutos em uma solução com fungicida a base de sulfato de cobre (calda bordalesa) na concentração de $4 \mathrm{~g}$ para 10L de água, para imediatamente serem transplantadas para os vasos.

O delineamento experimental utilizado foi de blocos ao acaso com quatro repetições. Foram utilizados vasos de polietileno com capacidade para $5 \mathrm{dm}^{3}$ de substrato. Cada parcela foi constituída por cinco vasos com uma planta/vaso. Em cada um dos sistemas de cultivo foram avaliados frutos de 20 plantas de cada cultivar ao longo de todo o ciclo da cultura.

Adotou-se como padrão de colheita, frutos com mais de $80 \%$ da superfície com a coloração vermelho-intensa. Os frutos para as análises laboratoriais foram colhidos no mês de outubro, período equivalente ao pico de produção da cultura na região, sendo selecionados para armazenamento e congelamento aqueles com massa superior a $12 \mathrm{~g}$.

O substrato utilizado no sistema orgânico foi composto por subsolo e compostagem orgânica (resíduo de palhada de milho e esterco bovino) na proporção de 2:1, respectivamente. Já o substrato utilizado para o sistema convencional foi baseado de acordo com o recomendado para a cultura, ou seja, subsolo e fertilizante NPK (8-28-16), equivalente a $600 \mathrm{~kg} \mathrm{ha}^{-1}$.

O controle fitossanitário no sistema orgânico foi realizado por meio de pulverizações quinzenais com óleo de Nin (Azadirach) a 2\%. O controle preventivo de doenças fúngicas foi realizado mediante aplicações de calda bordaleza a 2,5\% a cada 15 dias. No sistema convencional, o controle fitossanitário foi realizado por meio de pulverizações quinzenais com Acephate e Abamectin para controle de pulgões e ácaros, respectivamente e de Iprodione para o controle preventivo de doenças fúngicas, conforme recomendado para a cultura do morangueiro.

A reposição nutricional de cobertura no sistema orgânico foi realizada por meio de aplicações de super magro na concentração de $5 \%$ a cada 30 dias. No sistema de cultivo convencional a adubação de cobertura a base de nitrogênio foi realizada por meio de pulverizações quinzenais com uréia a $0,5 \%$. A cada 30 dias foram aplicados boro e zinco via foliar nas concentrações de $1 \%$ e $3 \%$, respectivamente.

A caracterização química foi feita com uma amostra de aproximadamente 500 gramas de frutos, realizada por meio das seguintes determinações: sólidos solúveis (SS) medido em graus Brix e determinado em refratômetro de bancada marca Optech modelo RMT; acidez titulável (AT) determinada por método titulométrico e expressa em porcentagem de ácido cítrico (Normas Analíticas de Instituto Adolfo Lutz, 1985); relação (SS/AT); umidade, determinada por diferença de peso após secagem em estufa de circulação de ar à $70^{\circ} \mathrm{C}$ até peso constante; açúcares totais e açúcares redutores determinados pelo método titulométrico LaneEynon e expressos em porcentagem.

Os resultados foram submetidos à análise de variância utilizando o software Sisvar 5.0 e as diferenças entre as médias dos tratamentos foram comparadas pelo Teste de Tukey $(\mathrm{p}<0,05)$. 


\section{Resultados e discussão}

Por meio dos resultados obtidos mediante as caracterizações químicas dos frutos de morango produzidos em sistemas orgânico e convencional, pode-se afirmar que em relação ao teor de sólidos solúveis (SS) a cultivar Sweet Charlie apresentou maior valor, sendo estatisticamente superior a Camarosa no sistema convencional. No entanto, para o sistema orgânico observou-se um efeito contrário, onde a cultivar Camarosa foi significativamente superior a Sweet Charlie para a característica analisada (Tabelas 1 e 2).

Tabela 1. Médias dos teores de sólidos solúveis (SS), acidez titulável (AT) e ratio (SS/AT) de cultivares de morango em diferentes sistemas de cultivo.

\begin{tabular}{ccccccc}
\hline \multirow{2}{*}{ Cultivar } & \multicolumn{5}{c}{ Sistema de Produção } \\
\cline { 2 - 6 } & \multicolumn{5}{c}{ Convencional } & \multicolumn{3}{c}{ Orgânico } \\
\cline { 2 - 6 } & SS (Brix $\mathbf{0})$ & AT (\%) & SS/AT & SS (Brix $\mathbf{0})$ & AT (\%) & SS/AT \\
\hline Camarosa & $8,10 \mathrm{Bb}^{*}$ & $90,41 \mathrm{Ba}$ & $8,96 \mathrm{Bb}$ & $9,50 \mathrm{Aa}$ & $95,12 \mathrm{Aa}$ & $9,99 \mathrm{Aa}$ \\
Sweet Charlie & $9,43 \mathrm{Aa}$ & $91,54 \mathrm{Aa}$ & $10,30 \mathrm{Aa}$ & $8,18 \mathrm{Bb}$ & $84,10 \mathrm{Bb}$ & $9,65 \mathrm{Ba}$ \\
\hline
\end{tabular}

*Médias seguidas pela mesma letra maiúscula na linha e minúscula na coluna não diferem estatisticamente pelo teste de $\mathrm{T}$ b/ukey $(\mathrm{p}<0,05)$.

Tabela 2. Médias dos teores de umidade, açúcares redutores e açúcares totais de cultivares de morango em diferentes sistemas de cultivo.

\begin{tabular}{|c|c|c|c|c|c|c|}
\hline \multirow{3}{*}{ Cultivar } & \multicolumn{6}{|c|}{ Sistema de Produção } \\
\hline & \multicolumn{3}{|c|}{ Convencional } & \multicolumn{3}{|c|}{ Orgânico } \\
\hline & $\begin{array}{c}\text { Umidade } \\
(\%)\end{array}$ & $\begin{array}{c}\text { Açúcares } \\
\text { redutores } \\
\text { (\%) }\end{array}$ & $\begin{array}{c}\text { Açúcares } \\
\text { totais } \\
(\%)\end{array}$ & $\begin{array}{c}\text { Umidade } \\
(\%)\end{array}$ & $\begin{array}{c}\text { Açúcares } \\
\text { redutores } \\
\qquad(\%)\end{array}$ & $\begin{array}{c}\text { Açúcares totais } \\
(\%)\end{array}$ \\
\hline Camarosa & $89,14 \mathrm{Aa}^{*}$ & $4,93 \mathrm{Aa}$ & $5,65 \mathrm{Aa}$ & $89,01 \mathrm{Aa}$ & $4,87 \mathrm{Aa}$ & $5,92 \mathrm{Aa}$ \\
\hline Sweet Charlie & $90,38 \mathrm{Bb}$ & $5,56 \mathrm{Aa}$ & $6,48 \mathrm{Aa}$ & $88,92 \mathrm{Aa}$ & 4,97Aa & $5,16 \mathrm{Ba}$ \\
\hline
\end{tabular}

*Médias seguidas pela mesma letra maiúscula na linha e minúscula na coluna não diferem estatisticamente pelo teste de Tukey $(\mathrm{p}<0,05)$.

Este comportamento diferenciado entre as cultivares nos dois sistemas ocorre em função da interação genótipos x ambientes, conforme descrito por Darolt (2003). Rissini et al. (2005) também verificaram a utilização de adubação orgânica em complementação à adubação química em quatro cultivares de morango (Sweet Charlie, Camarosa, Dover e Oso Grande) e observaram que houve um incremento considerável no teor de sólidos solúveis dos frutos.

Abu-Zahra et al. (2007) em experimento com frutos de morango da cultivar Honor, na região nordeste do Vale do Jordão (Israel), concluíram que o cultivo orgânico tende a produzir frutos com maiores teores de sólidos solúveis totais. Resultado semelhante também foi obtido por Krolow et al. (2007) que verificaram um aumento de 16,1 \% no teor de sólidos solúveis totais com a cultivar Aromas sob manejo orgânico, quando comparado ao manejo convencional.

Quanto à porcentagem de acidez titulável (AT), não foi verificada diferença estatística entre as cultivares no sistema convencional. Porém, para o sistema orgânico, a cultivar Camarosa apresentou 
valores superiores, diferindo significativamente da Sweet Charlie. Krolow et al. (2007) observaram que o cultivo de morango no sistema convencional proporcionou um incremento de 5,2\% na acidez total titulável em frutos da cultivar Aromas, quando comparado ao sistema orgânico.

Considerandoa relação SS/AT, queé determinante da qualidade comercial dos frutos, a cultivar Sweet Charlie foi significativamente superior à Camarosa no sistema convencional. Já no sistema orgânico, as cultivares não apresentaram diferenças significativas entre si.

Para as características apresentadas na Tabela 1 (sólidos solúveis, acidez titulável e a relação sólidos solúveis/acidez titulável) observou-se, de modo geral, um comportamento diferenciado e oposto entre as cultivares. No sistema convencional, a cultivar Sweet Chalie apresentou valores mais elevados quando comparada a cultivar Camarosa. Em contrapartida, no sistema orgânico a cultivar Camarosa foi a que apresentou maiores valores para as características avaliadas. Estes resultados demonstram haver interação entre as cultivares e o ambiente, estando de acordo com o observado por Darolt (2003), que menciona o efeito do sistema (orgânico e convencional) nas propriedades químicas do morango.

A umidade determina a porcentagem de água presente na polpa do fruto. Nesse sentido, a cultivar Camarosa apresentou menor valor no sistema convencional quando comparada a Sweet Charlie, e para o sistema orgânico as cultivares não diferiram estatisticamente (Tabela 2).

$\mathrm{O}$ teor de açúcares redutores (glicose e frutose) e o teor de açúcares totais não diferiram estatisticamente entre as duas cultivares, tanto no sistema convencional quanto no sistema orgânico (Tabela 2).

\section{Conclusão}

Para a maioria das características físico-químicas avaliadas foi verificada interação entre as cultivares e os sistemas de cultivo. Dessa forma, com base nos resultados obtidos, a cultivar Sweet Charlie é a mais indicada para o cultivo em sistema convencional enquanto que a cultivar Camarosa apresenta frutos com melhor qualidade quando cultivada em sistema orgânico.

\section{Referências}

ABU-ZAHRA, T.R.; AL-ISMAIL, K.; SHATAT, F. Effect of organic and conventional systems on fruit quality of strawberry (Fragaria $\mathrm{x}$ ananassa Duch) grown under plastic house conditions in the Jordan Valley. Acta Horticulturae, Leuven, n.741, p.159-171, abr. 2007.

ANTUNES, L.E.C.; REISSER JÚNIOR, C. Produção de morangos. Jornal da Fruta, Lages, v.15, n.191, p.22-24, 2007.

DAROLT, M. R. Morango: sistema orgânico apresenta viabilidade técnica, econômica e ecológica. 2001. Disponível em: <http://www.planetaorganico.com.br/ darmorang.htm>. Acesso em: 2 mar. 2007.

DAROLT, M. R. Comparação da qualidade do alimento orgânico com o convencional. In: STRIGHETA, P. C.; MUNIZ, J. N. (Ed.). Alimentos Orgânicos: produção, tecnologia e certificação. Viçosa: UFV, 2003. p. 289312 .

DIAS, M. S. C.; RIBEIRO JÚNIOR, P. M.; SILVA, M. S.; SANTOS, L. O.; CANUTO, R. S.; CASTRO, M. V.; COSTA, S. M. Caracterização físico-química de morangos cultivados na região norte de Minas Gerais . In: CONGRESSO BRASILEIRO DE FRUTICULTURA, 17., 2002, Belém. Anais... Belém: Sociedade Brasileira de Fruticultura, 2002.

INSTITUTO ADOLFO LUTZ. Normas analíticas do instituto Adolfo Lutz: métodos químicos e físicos para análises de alimentos. São Paulo: IAL, 1985. 183 p.

KROLOW, A.C.; SCHWENGBER, J.; FERRI, N. Avaliações físicas e químicas de morangos cv. Aromas produzidos em sistema orgânico e convencional. Revista Brasileira de Agroecologia, v.2, n.2, out.2007. 
RISSINI, A.L.L.; FARIA, M.V.; RESENDE，J.T.V.; BARBOSA, M.R.; SCHIMILOSKI, S.; FARIA, C.M.D.R.; MARCHESE, A.; BORTOLI, C.; DENEGA, S. Sólidos solúveis de frutos de cultivares de morango sob diferentes doses de fertilizante orgânico em cultivo protegido (compact disc). In: CONGRESSO BRASILEIRO DE OLERICULTURA, 2005, Fortaleza. Resumos... Fortaleza:SOB, 2005.

SAMINÊZ, T. C. O. Agricultura orgânica: mercado em expansão. Revista Brasileira Agropecuária, Rio de Janeiro, ano. 1, n. 9, p. 43, 2000.
SANHUEZA, R. M. V. Produção integrada de frutas. In: CONGRESSO BRASILEIRO DE FRUTICULTURA, 16., 2000, Fortaleza. Anais... Fortaleza: SBF/Embrapa Agroindústria Tropical, 2000. (CD-ROM).

SCHERER, E. E.; VERONA, L. A. F.; SIGNOR, G.; VARGAS, R.; INNOCENTE, B. Produção agroecológica de morangos no Oeste Catarinense. Agropecuária Catarinense, Florianópolis, v. 16, n. 1, p. 20-24, 2003.

WILLIAMS. C. M. Nutritional quality of organic food: shades of grey or shades of green? Proceedings of the Nutrition Society, London, v. 61, n. 1, p. 19-24, 2002. 